\title{
Vector states above open charm threshold at BESIII
}

\author{
Zhi-yong Wang, a \\ ${ }^{1}$ BESIII Collaboration
}

\begin{abstract}
Based on the the large XYZ data sample collected at BESIII detector, we study the vector states above charm threshold, including the traditional charmonium $\psi$ and charmonium-like states, $Y$ in many decay modes. For example, $e^{+} e^{-} \rightarrow$ $h_{c}, \pi^{+} \pi^{-} J / \psi, \psi(3686), e^{+} e^{-} \rightarrow \eta\left(\eta^{\prime}\right) J / \psi$. Many charmonium and charmonium-like candidates are found, but some of them need to be confirmed further with much larger data.
\end{abstract}

\section{Introduction}

The process $\pi^{+} \pi^{-} J / \psi$ at center-of-mass (c.m.) energies between 3.8 and $5.0 \mathrm{GeV}$ was first studied by the BABAR experiment using an initial-state-radiation (ISR) technique, and a new structure, the $Y(4260)$, was reported with a mass around $4.26 \mathrm{GeV} / \mathrm{c}^{2}$. This observation was immediately confirmed by the CLEO [1] and Belle experiments [2] in the same process. In addition, the Belle experiment reported an accumulation of events at around $4 \mathrm{GeV}$, which was called $Y(4008)$ later. Although the $Y(4008)$ state is still controversial--a new measurement by the BABAR experiment does not confirm it [3], while an updated measurement by the Belle experiment still supports its existence--the observation of the $Y$ states has stimulated substantial theoretical discussions on their nature $[4,5]$.

Being produced in $e^{+} e^{-}$annihilation, the Y states have quantum numbers $J^{P C}=1^{--}$. However, unlike the known $1^{--}$charmonium states in the same mass range, such as $\psi(4040), \psi(4160)$, and $\psi(4415)$, which decay predominantly into open charm final states $\left[D^{(*)} \bar{D}^{(*)}\right]$, the $Y$ states show strong coupling to hidden-charm final states [6]. Furthermore, the observation of the states $Y(4360)$ and $Y(4660)$ in $e^{+} e^{-} \rightarrow \pi^{+} \pi^{-} \psi(3686)$ [7], together with the newly observed resonant structures in $e^{+} e^{-} \rightarrow$ $\omega \chi_{c J}(\mathrm{~J}=0,1,2)$ and $e^{+} e^{-} \rightarrow \pi^{+} \pi^{-} h_{c}$, overpopulates the vector charmonium spectrum predicted by potential models [8]. All of this indicates that the $\mathrm{Y}$ states may not be conventional charmonium states, and they are good candidates for new types of exotic particles, such as hybrids, tetraquarks, or meson molecules

We search the Y states via $e^{+} e^{-} \rightarrow \pi \pi J / \psi, K \bar{K} J / \psi, \pi \pi \psi(3686), \pi^{+} \pi^{-} h_{c}, \pi^{+} \pi^{-} X(3823), e^{+} e^{-} \rightarrow$ $\omega \chi_{c J}(J=0,1,2)$, the radiative decay of $e^{+} e^{-} \rightarrow \pi^{+} D^{0} D^{*-}$, and the radiative decay of $e^{+} e^{-} \rightarrow$ $\gamma X(3872)$. We observed some more complicate structure than before.

ae-mail: wangzy@ihep.ac.cn 


\section{$2 e^{+} e^{-} \rightarrow \operatorname{PPc} \bar{c}\left(\mathbf{P}=\pi, K, c \bar{c}=J / \psi, h_{c}, \psi(3686)\right)$}

$2.1 e^{+} e^{-} \rightarrow \pi \pi\left(\pi^{+} \pi^{-}, \pi^{0} \pi^{0}\right) J / \psi$

The $J / \psi$ is constructed by leptonic pair, $e^{+} e^{-}$and $\mu^{+} \mu^{-}$final states. The cross section of $e^{+} e^{-} \rightarrow$ $\pi^{+} \pi^{-} J / \psi$ at a certain $e^{+} e^{-}$c.m. energy $\sqrt{s}$ is calculated using

$$
\sigma(\sqrt{s})=\frac{N^{\text {sig }}}{\mathcal{L}(1+\delta) \epsilon \mathcal{B}}
$$

where $N^{\text {sig }}$ is the number of signal events, $\mathcal{L}_{\text {int }}$ is the integrated luminosity of data, $(1+\delta)$ is the ISR correction factor, $\epsilon$ is the detection efficiency, and $\mathcal{B}$ is the branching fraction of $J / \psi \rightarrow \ell^{+} \ell^{-}$. Figure 1 (left) and (middle) show the measured cross section from both the XYZ data and scan data. To study the possible resonant structures in the $e^{+} e^{-} \rightarrow \pi^{+} \pi^{-} J / \psi$ process, a binned maximum likelihood fit is performed simultaneously to the measured cross section $\sigma(\sqrt{s})$ of the XYZ data with Gaussian uncertainties and the scan data with Poisson uncertainties. The PDF is parameterized as the coherent sum of three Breit-Wigner (BW) functions, together with an incoherent $\psi(3770)$ component which accounts for the decay of $\psi(3770) \rightarrow \pi^{+} \pi^{-} J / \psi$, with $\psi(3770)$ mass and width fixed to the Particle Data Group (PDG) values.
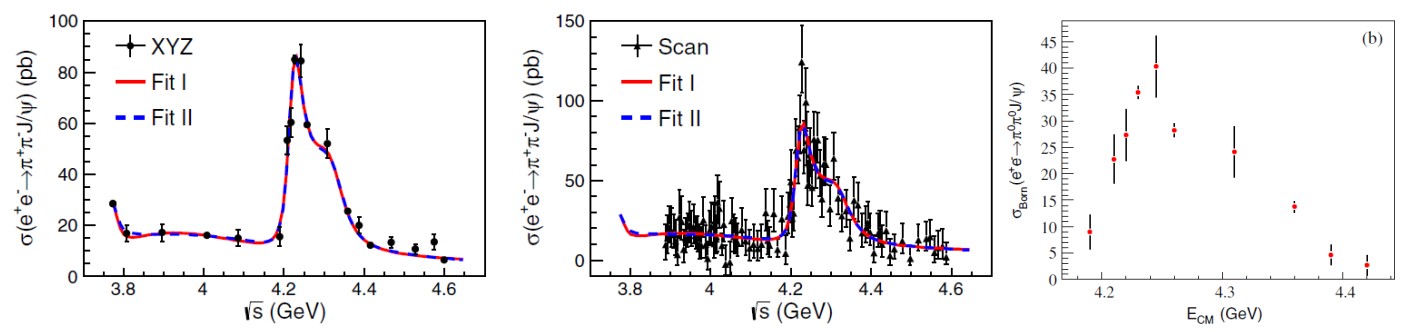

Figure 1. Measured cross section $\sigma\left(e^{+} e^{-} \rightarrow \pi^{+} \pi^{-} J / \psi\right)$ and simultaneous fit to the XYZ data (left) and scan data (middle) with the coherent sum of three Breit-Wigner functions (red solid curves) and the coherent sum of an exponential continuum and two Breit-Wigner functions (blue dashed curves). (right) $\sigma_{\text {Born }}\left(e^{+} e^{-} \rightarrow \pi^{0} \pi^{0} J / \psi\right)$ as a function of $E_{\text {c.m. }}(\sqrt{s})$ with error bars that are statistical only. Dots with error bars are data.

Similar to the $e^{+} e^{-} \rightarrow \pi^{+} \pi^{-} J / \psi$, we also study its isospin neutral channel $e^{+} e^{-} \rightarrow \pi^{0} \pi^{0} J / \psi$. we not only observed a neutral charmonium-like structure, $Z_{c}(3900)^{0}$, but also measured its Born cross section, $\sigma^{B}\left(e^{+} e^{-} \rightarrow \pi^{0} \pi^{0} J / \psi\right)$. Figure 1 (right) shows the Born cross section as functions of $E_{\text {c.m. }}(\sqrt{s})$ with error bars that are statistical only. we are hard to determine whether there are any structures around $4.26 \mathrm{GeV}$.

The $e^{+} e^{-} \rightarrow K \bar{K} J / \psi$ is also studied using $4.7 \mathrm{fb}^{-1}$ of data with energies ranging from 4:189 to 4:600 GeV. Figure 2 shows the Born cross section as function of $E_{\text {c.m. }}$ for $e^{+} e^{-} \rightarrow$ $K^{+} K^{-} J / \psi, K_{S}^{0} K_{S}^{0} J / \psi$. Compared with Fig. 1, one finds that it is obviously different from that for charged channel. The results are inconclusive as to whether $Y(4260)$ decays to $K \bar{K} J / \psi$. In addition, there is evidence for an enhancement in $e^{+} e^{-} \rightarrow K \bar{K} J / \psi$ in the higher mass region. More data and additional analyses will be needed to investigate the nature of this structure.

$2.2 e^{+} e^{-} \rightarrow \pi^{+} \pi^{-} h_{c}$

we present a follow-up study of $e^{+} e^{-} \rightarrow \pi^{+} \pi^{-} h_{c}$ at c.m. energies from 3.896 to $4.600 \mathrm{GeV}$ using data samples taken at 79 energy points. The $h_{c}$ is reconstructed via its electric-dipole transition $h_{c} \rightarrow \gamma \eta_{c}$ 


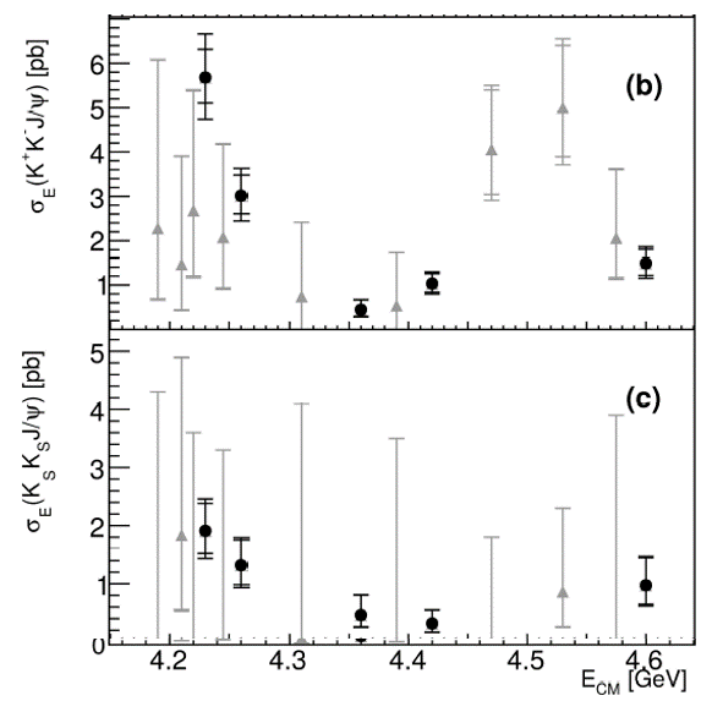

Figure 2. he gray triangular points are for the data with lower luminosities, while the black circular points are for the data with higher luminosities. Errors are shown with both the statistical error and the combined statistical and systematic errors.

with $\eta_{c}$ decaying to 16 exclusive hadronic final states. Figure 3 shows the Dressed cross section (including vacuum polarization effect). A maximum likelihood method is used to fit the dressed cross sections to determine the parameters of the resonant structures. The likelihood is constructed taking the fluctuations of the number of signal and background events into account (the definition is described in the Supplemental Material). Assuming that the $\pi^{+} \pi^{-} h_{c}$ signal comes mnfrom two resonances, the cross section is parametrized as the coherent sum of two constant width relativistic BW functions. We obtain $M=\left(4218.4_{-4.5}^{+5.5} \pm 0.9\right) \mathrm{MeV} / \mathrm{c}^{2}, \Gamma=\left(66.0_{-8.3}^{+12.3} \pm 0.4\right) \mathrm{MeV}$, and $\left(\Gamma_{e e} \mathcal{B}\right)=\left(4.6_{-1.4}^{+2.9} \pm 0.8\right) \mathrm{eV}$ for $Y(4220)$, and $M=\left(4391.5_{-6.8}^{+6.3} \pm 1.0\right) \mathrm{MeV} / \mathrm{c}^{2}, \Gamma=\left(139.5_{-20.6}^{+16.2} \pm 0.6\right) \mathrm{MeV}$, and $\left(\Gamma_{e e} \mathcal{B}\right)=\left(11.6_{-4.4}^{+5.0} \pm 1.9\right)$ $\mathrm{eV}$ for $Y(4390)$, with a relative phase of $\phi=\left(3.1_{-0.9}^{+0.7} \pm 0.2\right) \mathrm{rad}$.

$2.3 e^{+} e^{-} \rightarrow \pi \pi\left(\pi^{+} \pi^{-}, \pi^{0} \pi^{0}\right) \psi(3686)$

In these decays, the $\psi(3686)$ is constructed by $\pi^{+} \pi^{-} J / \psi$ with $J / \psi$ decaying to leptonic pairs $\left(e^{+} e^{-}, \mu^{+} \mu^{-}\right)$. The comparison of the combined Born cross section of $e^{+} e^{-} \rightarrow \pi^{+} \pi^{-} \psi(3686)$ with those from previous experimental results is shown in Fig. 4 (left). The results are consistent with former experiments, and have much improved precision, while the comparison of the Born cross section of $e^{+} e^{-} \rightarrow \pi^{0} \pi^{0} \psi(3686)$ to that of $e^{+} e^{-} \rightarrow \pi^{+} \pi^{-} \psi(3686)$ for the data samples with large luminosities is shown in Fig. 4 (right). The latter are found to be half of those for $e^{+} e^{-} \rightarrow \pi^{+} \pi^{-} \psi(3686)$ within uncertainties, consistent with the expectation from isospin symmetry.

$$
3 e^{+} e^{-} \rightarrow \pi^{+} \pi^{-} X(3823)
$$

the Belle Collaboration reported evidence for a narrow resonance $\mathrm{X}(3823) \rightarrow \gamma \chi_{c 1}$ in $\mathrm{B}$ meson decays with $3.8 \sigma$ significance and mass $3823.1 \pm 1.8 \pm 0.7 \mathrm{MeV} / \mathrm{c}^{2}$, and suggested that this is a good candidate for the $1^{3} D_{2}$ charmonium state [9]. The $\mathrm{X}(3823)$ candidates are reconstructed in their $\gamma \chi_{c 1}$ and $\gamma \chi_{c 2}$ 


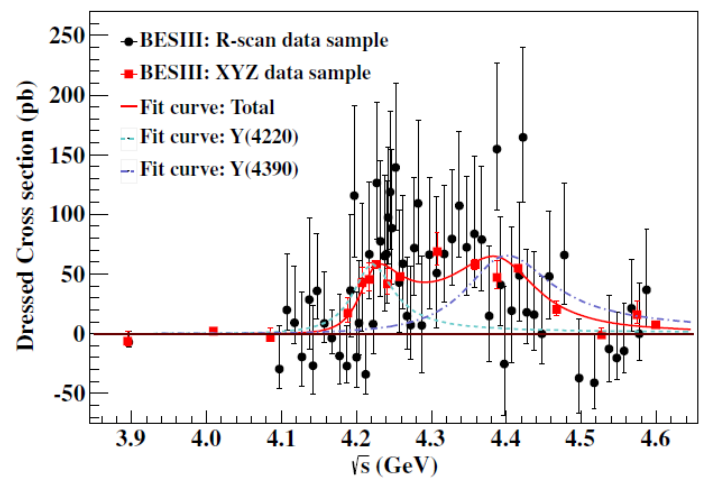

Figure 3. Fit to the dressed cross section of $e^{+} e^{-} \rightarrow \pi^{+} \pi^{-} h_{c}$ with the coherent sum of two BW functions (solid curve). The dash (dash-dot) curve shows the contribution from the two structures $Y(4220)$ [Y(4390)]. The dots with error bars are the cross sections for the R-scan data sample, the squares with error bars are the cross sections for the XYZ data sample. Here the error bars are statistical uncertainty only.
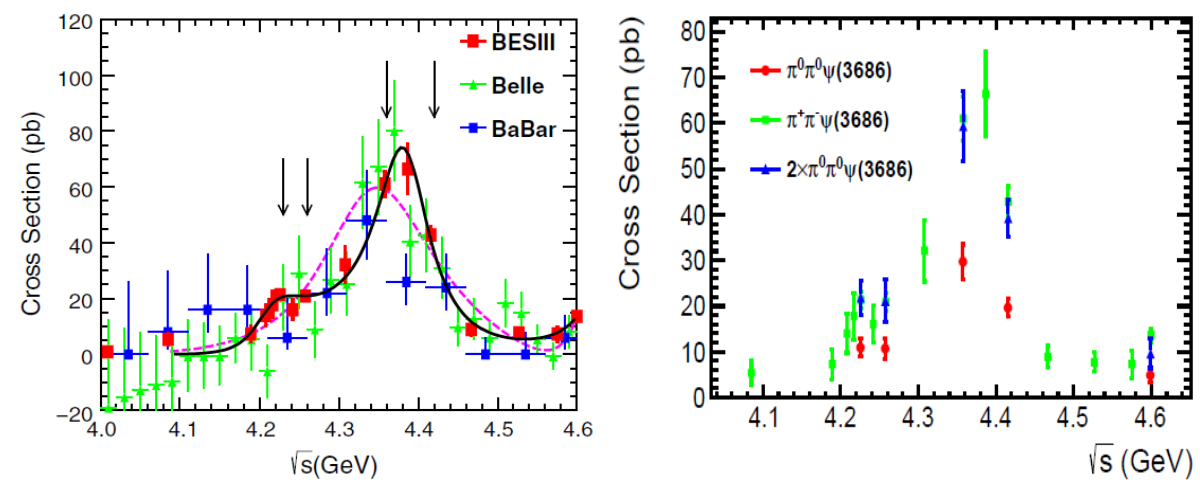

Figure 4. (left) Born cross section of . The dots are the results obtained in this analysis, the triangles and squares are from BELLE and BABAR latest updated results, respectively. The solid curve is the fit to BESIII results with the coherent sum of three Breit-Wigner functions. The dashed curve (pink) is the fit to BESIII results with the coherent sum of two Breit-Wigner functions without the $Y(4220)$ hypothesis. The arrows mark the locations of four energy points with large luminosities. (right) Born cross section of $e^{+} e^{-} \rightarrow \pi^{0} \pi^{0} \psi(3686)$ at $\sqrt{s}=4.226$, $4.258,4.358,4.416,4.600 \mathrm{GeV}$, respectively. The dots (red) are the results obtained in this analysis, the triangles (green) are twice of our results and squares (blue) are the Born cross section of $e^{+} e^{-} \rightarrow \pi^{+} \pi^{-} \psi(3686)$ from BESIII.

decay modes, with $\chi_{c 1,2} \rightarrow \gamma J / \psi$ and $J / \psi \rightarrow \ell^{+} \ell^{-}(l=e$ or $\mu)$. We performed a search for the production of the $X(3823)$ state using $4.67 \mathrm{fb}^{-1}$ data at center of mass (c.m.) energies that range from $\sqrt{s}=4.19$ to $4.60 \mathrm{GeV}$. Figure 5 (left) shows the $\pi^{+} \pi^{-}$recoil mass with $\gamma \chi_{c 1}$ mass region. clear $X(3823)$ signal is seen. An unbinned maximum likelihood fit to the $M_{\text {recoil }}\left(\pi^{+} \pi^{-}\right)$distribution is performed to extract the $\mathrm{X}(3823)$ signal parameters. Figure 5 (right) shows the energy-dependent cross sections of $e^{+} e^{-} \rightarrow \pi^{+} \pi^{-} X(3823)$ which is fitted with the $Y(4360)$ shape or the $\psi(4415)$ shape 
with their resonance parameters fixed to the PDG values. We accept both the $Y(4360)$ and the $\psi(4415)$ hypotheses according to the Kolmogorov test.
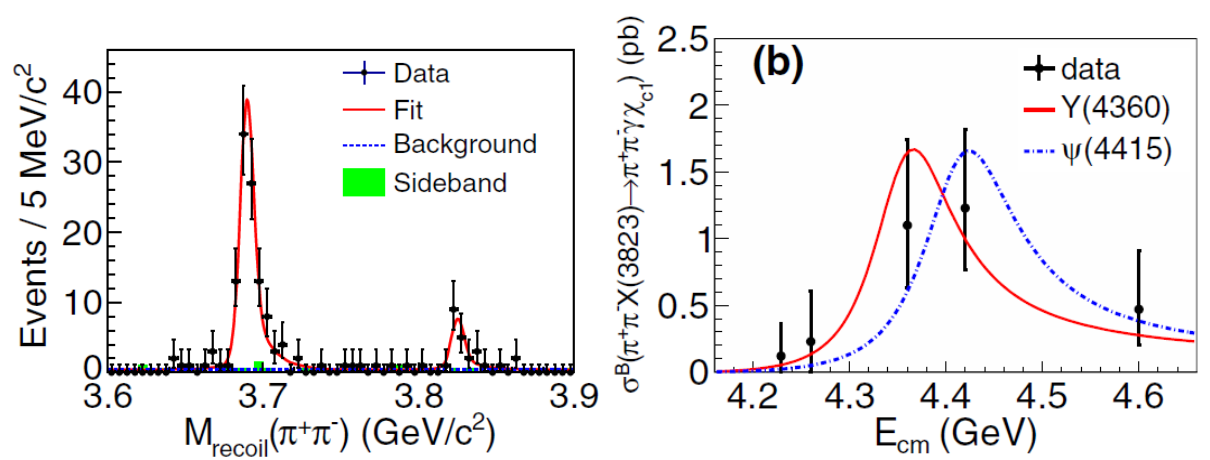

Figure 5. (left) Simultaneous fit to the distribution of $\gamma \chi_{c 1}$ events, respectively. Dots with error bars are data. (right) fit to the energy-dependent cross section of $\sigma^{B}\left[e^{+} e^{-} \rightarrow \pi^{+} \pi^{-} X(3823)\right] \mathcal{B}\left[X(3823) \rightarrow \gamma \chi_{c 1}\right]$ with the $\mathrm{Y}(4360)$ (red solid curve) and the $\psi(4415)$ (blue dashed curve) line shapes.

\section{$4 e^{+} e^{-} \rightarrow \gamma X(3872)$}

With the data samples collected at four energy points ranging from $\sqrt{s}=4.009$ to $4.420 \mathrm{GeV}$, we present a first observation of $e^{+} e^{-} \rightarrow \gamma X(3872) \rightarrow \gamma \pi^{+} \pi^{-} J / \psi, J / \psi \rightarrow \ell^{+} \ell^{-}$). Figure 6 (left) shows the $\pi^{+} \pi^{-} J / \psi$ invariant mass distributions by sum of four energy points. Clear $X(3872)$ signal is seen. Figure 6 (right) shows the energy-dependent cross section which is fitted with a Y(4260) resonance (parameters fixed to PDG values), a linear continuum, or a E1-transition phase space term. The $Y(4260)$ resonance describes the data better than the other two options. However, we can not draw a conclusion that $Y(4260)$ is bound to decay to $\gamma X(3872)$ due to the poor statistics.
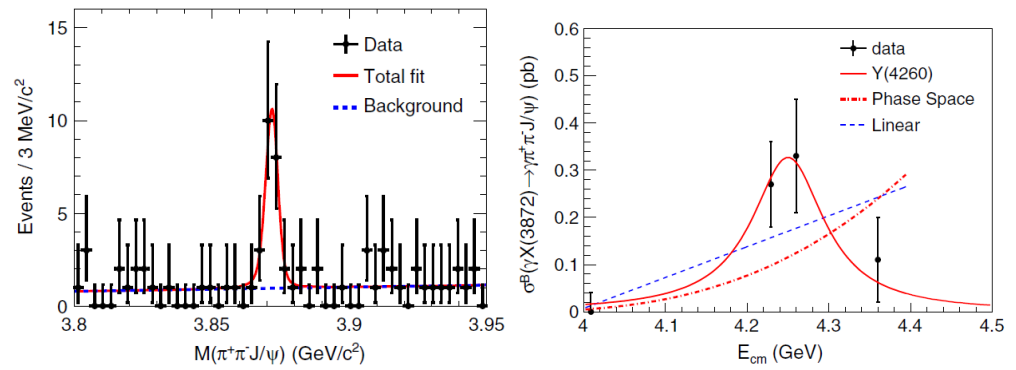

Figure 6. (left) Fit of the $M_{\pi^{+} \pi^{-} J / \psi}$ distribution with a MC simulated histogram convolved with a Gaussian function for signal and a linear background function. Dots with error bars are data, the red curve shows the total fit result, while the blue dashed curve shows the background contribution. (right) The fit to $\sigma^{B}\left[e^{+} e^{-} \rightarrow\right.$ $\gamma X(3872)] \times \mathcal{B}\left[X(3872) \rightarrow \pi^{+} \pi^{-} J / \psi\right]$ with a Y(4260) resonance (red solid curve), a linear continuum (blue dashed curve), or a E1-transition phase space term (red dotted-dashed curve). 


$$
5 e^{+} e^{-} \rightarrow \omega \chi_{c J}(J=0,1,2)
$$

We performed a study of $e^{+} e^{-} \rightarrow \omega \chi_{c J}(J=0,1,2)$ based on the data samples at fourteen center of mass energy points in the range $\sqrt{s}=4.21-4.59 \mathrm{GeV}$. In the analysis, the $\omega$ meson is reconstructed via its $\pi^{+} \pi^{-} \pi^{0}$ decay mode, the $\chi_{c 0}$ state is via $\pi^{+} \pi^{-}$and $K^{+} K^{-}$decays, and the $\chi_{c 1,2}$ states are via $\chi_{c 1,2} \rightarrow \gamma J / \psi, J / \psi \rightarrow \ell^{+} \ell^{-}$. In Fig. 7, we compare the line shapes of the Born cross sections for $e^{+} e^{-} \rightarrow \omega \chi_{c J}$, where the Born cross sections at $\sqrt{s}<4.4 \mathrm{GeV}$ are from the previous observation [? ]. Enhancements can be seen in the line shapes; in the following, we try to fit line shapes. The cross section of $e^{+} e^{-} \rightarrow \omega \chi_{c 0}$ with the addition of higher energy points is refitted with a phase-space modified BW function, and the fit results for the structure parameters are $\Gamma_{e e} \mathcal{B}\left(\omega \chi_{c 0}\right)=(2.8 \pm 0.5 \pm 0.4)$ $\mathrm{eV}, M=(4226 \pm 8 \pm 6) \mathrm{MeV} / \mathrm{c}^{2}$, and $\Gamma_{t o t}=(39 \pm 12 \pm 2) \mathrm{MeV}$, which are almost unchanged. In the $e^{+} e^{-} \rightarrow \omega \chi_{c 2}$ cross section, an enhancement is seen around $4.416 \mathrm{GeV}$, so we use a coherent sum of the $\psi(4415) \mathrm{BW}$ function and a phase-space term to fit the cross section with fixing some parameters to known $\psi(4415)$ state. Two resolutions are obtained with the same fit quality. One is constructive, while the other is destructive.

\section{Search $Y(4140)$ via $e^{+} e^{-} \rightarrow \gamma \phi J / \psi$}

The CDF experiment first reported evidence for a new state called $Y(4140)$ in the decay $B^{+} \rightarrow$ $\phi J / \psi K^{+}$[11]. In a subsequent analysis, CDF claimed the observation of the $Y(4140)$ with a statistical significance greater than $5 \sigma$. However, the existence of the $Y(4140)$ was not confirmed by the Belle [12] or LHCb [13] Collaborations in the same process, nor by the Belle Collaboration in two-photon production. Recently, the CMS [14] and D0 [15] Collaborations reported on analyses of $B^{+} \rightarrow \phi J / \psi K^{+}$, where an accumulation of events is observed in the $\phi J / \psi$ invariant mass distribution, with resonance parameters consistent with those of the CDF measurement. The BABAR Collaboration also investigated the same decay mode, and found no evidence for the Y(4140) [16]. we present results of a search for $Y(4140)$ decays into $\phi J / \psi$ through the process $e^{+} e^{-} \rightarrow \gamma \phi J / \psi$ with data taken at $\mathrm{CM}$ energies of $\sqrt{s}=4.23,4.26$, and $4.36 \mathrm{GeV}$. The $\phi$ is tagged by $K^{+} K^{-}, K_{S}^{0} K_{S}^{0}$, and $\pi^{+} \pi^{-} \pi^{0}$ final state, respectively. The $J / \psi$ is tagged by leptonic pair, just like before. The invariant mass distributions of the $\phi J / \psi\left(\phi \rightarrow K^{+} K^{-}\right)$candidates by sum of four energy points after all event selection criteria have been applied are shown in Fig. 8. No $Y(4140)$ signal is observed. Also no $Y(4140)$ signal is found in the other final states, for example, $\phi \rightarrow K_{S}^{0} K_{S}^{0}, \pi^{+} \pi^{-} \pi^{0}$.

\section{$7 e^{+} e^{-} \rightarrow P J / \psi\left(P=\eta, \eta^{\prime}\right)$}

\section{$7.1 e^{+} e^{-} \rightarrow \eta J / \psi$}

Belle Collaboration observed the cross section as a function of c.m. energy for $e^{+} e^{-} \rightarrow \eta J / \psi$. They fit the line-shape with traditional charmonium states, $\psi(4040)$ and $\psi(4160)$, with interference [17]. However, the significance for these two resonances is low (see the Fig. 9 (left)). We performed a measurement of the Born cross sections of $e^{+} e^{-} \rightarrow \eta J / \psi$ at 17 c.m. energies from 3.810 to 4.600 $\mathrm{GeV}$. In our analysis, the $\eta$ are reconstructed in their two-photon decay mode and the $J / \psi$ via its decay into lepton pairs $\left(\ell^{+} \ell^{-}\right)$. Figure 9 (right) shows the comparison of the Born cross section of $e^{+} e^{-} \rightarrow \eta J / \psi$ to that previous measurement. One also notices that it is very different from that of $e^{+} e^{-} \rightarrow \pi^{+} \pi^{-} J / \psi$ according to Fig. 1 . This could indicate the existence of a rich spectrum of Y states in this energy region with different coupling strengths to the various decay modes. 


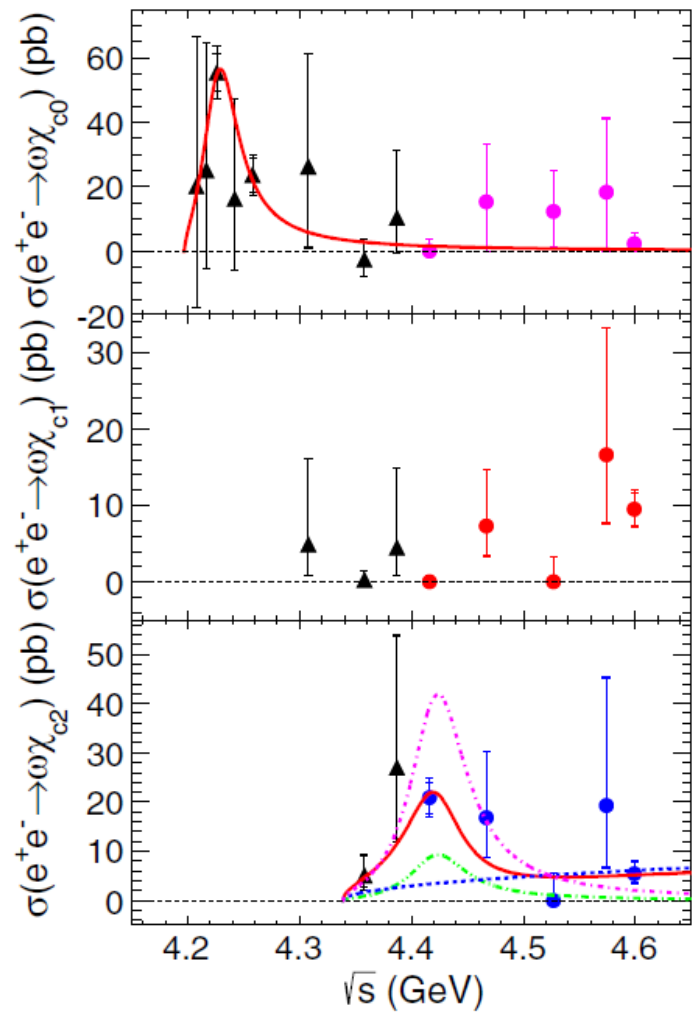

Figure 7. Measured Born cross section (center value) for $e^{+} e^{-} \rightarrow \omega \chi_{c J}(J=0,1,2)$ as a function of the center of mass energy. The top plot is for $e^{+} e^{-} \rightarrow \omega \chi_{c 0}$, the middle plot for $e^{+} e^{-} \rightarrow \omega \chi_{c 1}$, and the bottom plot for $e^{+} e^{-} \rightarrow \omega \chi_{c 2}$, where the smaller errors are statistical only and the larger errors are the quadratic sum of the statistical and systematic errors. The triangle black points are from previous observation, and others are from this analysis. The $e^{+} e^{-} \rightarrow \omega \chi_{c 0}$ is fitted with a resonance(solid curve) in the top plot. $e^{+} e^{-} \rightarrow \omega \chi_{c 2}$ is fitted with the coherent sum of the $\psi(4415) \mathrm{BW}$ function and a phase-space term. The solid curve shows the fit result, the blue dashed curve is the phase-space term, which is almost the same for the two solutions. The purple dash-dotted curve is the destructive solution and the green dash-double-dotted curve is the constructive one.

$7.2 e^{+} e^{-} \rightarrow \eta^{\prime} J / \psi$

To check the theoretical predictions [18] and to search for potential $\eta^{\prime} J / \psi$ transitions from charmonium and charmonium-like states, we measure the process $e^{+} e^{-} \rightarrow \eta^{\prime} J / \psi$ with the data taken at BESIII. The CLEO-c experiment searched for this process with data at c.m. energies $\sqrt{s}$ from 3.970 to $4.260 \mathrm{GeV}$ and did not observe the signal [19]. In the analysis, the $J / \psi$ is reconstructed through its decays into lepton pairs $J / \psi \rightarrow$, while the $\eta$ is reconstructed in two decay channels, $\eta^{\prime} \rightarrow \eta \pi^{+} \pi^{-}$ (with $\eta \rightarrow \gamma \gamma$ ) and $\eta^{\prime} \rightarrow \gamma \pi^{+} \pi^{-}$. Figure 10 shows the measured Born cross sections for $e^{+} e^{-} \rightarrow \eta^{\prime} J / \psi$ over the energy region studied in this work. Assuming that the $\eta^{\prime} J / \psi$ signals come from the $\psi(4160)$ decay, the cross section is fitted with a constant-width relativistic BW function.

Compared with the Born cross section of $e^{+} e^{-} \rightarrow \eta J / \psi$ mentioned above, the measured Born cross section of $e^{+} e^{-} \rightarrow \eta^{\prime} J / \psi$ is much smaller, which is in contradiction to the calculation in Ref. [18]. There are two possible reasons contributing to this discrepancy. The cross section of $e^{+} e^{-} \rightarrow \eta^{\prime} J / \psi$ 


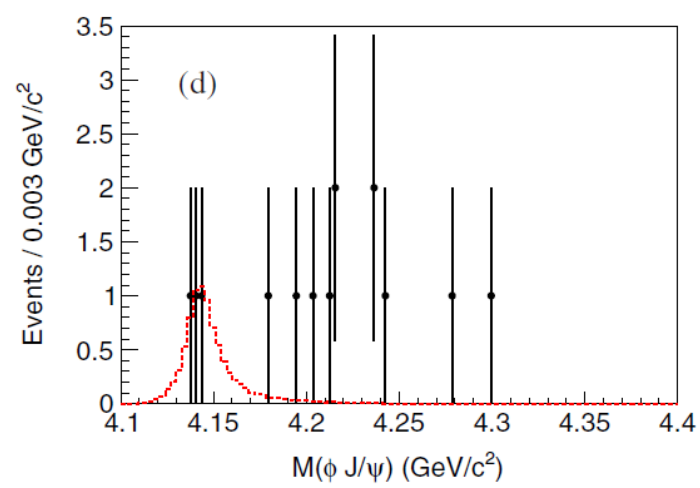

Figure 8. Distribution of $M_{\phi J / \psi}$ with $\phi$ decays to $K^{+} K^{-}$from the sum of three data samples. The red dashed histograms represent signal MC samples scaled to the measured upper limits.
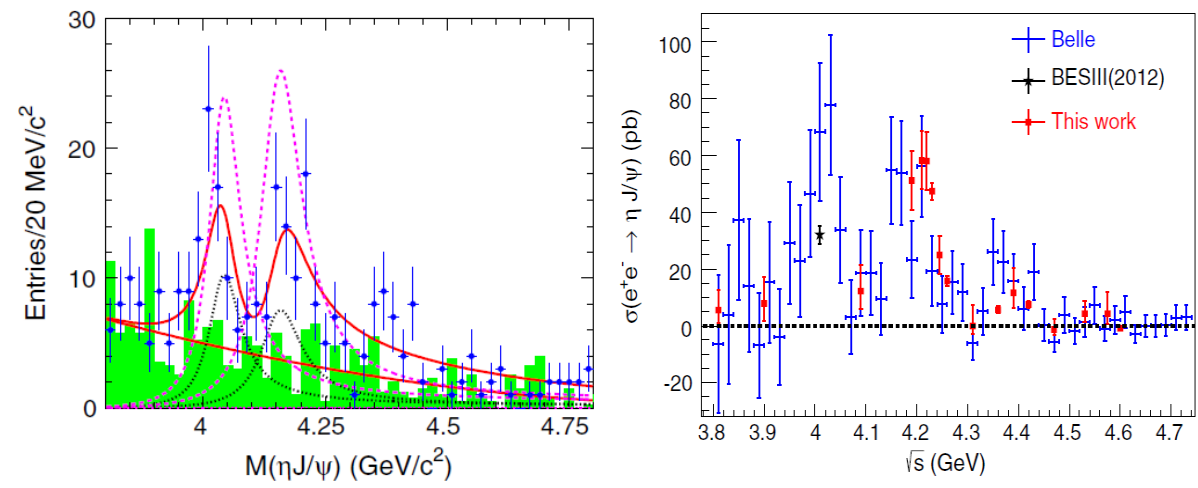

Figure 9. (left) A fit with $\psi(4040)$ and $\psi(4160)$ resonances to the Born cross section of $e^{+} e^{-} \rightarrow \eta J / \psi$ at Belle. (right) A comparison of the measured Born cross section of $e^{+} e^{-} \rightarrow \eta J / \psi$ to that of previous measurements. The blue dots are results from Belle, the black star dot is from BESIII (2012) and the red square dots are the results obtained in this work. The errors of the Belle data only include the statistical uncertainty. For the previously published BESIII results and our data, the errors reflect both statistical and systematical uncertainties.

is investigated at an order of $O\left(\alpha_{s}^{4}\right)$; therefore, higher order corrections might need to be considered; additionally, the proportion of gluonic admixture in $\eta^{\prime}$ needs to be further studied to make certain the contribution of a gluonium component on the results.

\section{Summary}

With the XYZ data collected at the BESIII detector, we search the vector state above charm threshold, including the traditional charmonium states and the charmonium-like state, $Y$. We try to search the new decay modes for these vector states. We observed some $\psi$ or new $Y$ candidates in $e^{+} e^{-} \rightarrow$ $\pi^{+} \pi^{-} h_{c}, \pi^{+} \pi^{-} J / \psi, \pi^{+} \pi^{-} \psi(3686)$ and $e^{+} e^{-} \rightarrow \omega \chi_{c J}$. We found that there are more complicate structure in $\pi^{+} \pi^{-} J / \psi$ line shape than previous measurement. We also find there are the similar structure in different line shapes. For example, $Y(4220)$ candidates can appear in $\pi^{+} \pi^{-} h_{c}, \pi^{+} \pi^{-} \psi(3686)$ and $\omega \chi_{c 0}$. 


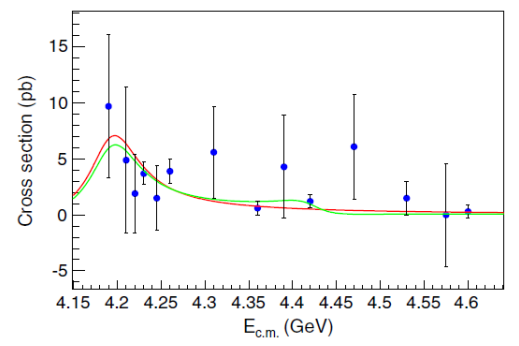

Figure 10. Fit to the Born cross section $e^{+} e^{-} \rightarrow \eta^{\prime} J / \psi$ with a $\psi(4160)$ resonance (red curve), or a coherent sum of $\psi(4160)$ and $\psi(4415)$ amplitudes (green curve).

However, we can not take it as the same states due to the poor statistics. We can not judge wether there is one, or more structures in some line shapes, either.

\section{References}

[1] Q. He et al. (CLEO Collaboration), Phys. Rev. D 74, 091104(R) (2006).

[2] C. Z. Yuan et al. (Belle Collaboration), Phys. Rev. Lett. 99, 182004 (2007).

[3] J. P. Lees et al. (BABAR Collaboration), Phys. Rev. D 86, 051102(R) (2012).

[4] N. Brambilla et al., Eur. Phys. J. C 71, 1534 (2011).

[5] H. X. Chen, W. Chen, X. Liu, and S. L. Zhu, Phys. Rep. 639, 1 (2016).

[6] X. H. Mo, G. Li, C. Z. Yuan, K. L. He, H. M. Hu, J. H. Hu, P. Wang, and Z. Y. Wang, Phys. Lett. B 640, 182 (2006).

[7] B. Aubert et al. (BABAR Collaboration), Phys. Rev. Lett. 98, 212001 (2007); X. L. Wang et al. (Belle Collaboration), Phys. Rev. Lett. 99, 142002 (2007); B. Aubert et al. (BABAR Collaboration), Phys. Rev. D 89, 111103 (2014); X. L. Wang et al. (Belle Collaboration), Phys. Rev. D 91, 112007 (2015).

[8] E. Eichten, K. Gottfried, T. Kinoshita, K. D. Lane, and T. M. Yan, Phys. Rev. D 17, 3090 (1978);

21, 203 (1980); S. Godfrey and N. Isgur, Phys. Rev. D 32, 189 (1985).

[9] V. Bhardwaj et al. (Belle Collaboration), Phys. Rev. Lett. 111, 032001 (2013).

[10] Ablikim et al. (BESIII Collaboration), Phys. Rev. Lett. 114, 092003 (2015).

[11] T. Aaltonen et al. (CDF Collaboration), Phys. Rev. Lett. 102, 242002 (2009).

[12] C. P. Shen et al. (Belle Collaboration),Phys. Rev. Lett. 104, 112004 (2010).

[13] R. Aaij et al. (LHCb Collaboration), Phys. Rev. D 85, 091103 (2012).

[14] S. Chatrchyan et al. (CMS Collaboration), Phys. Lett. B 734, 261 (2014).

[15] V. M. Abazov et al. (D0 Collaboration), Phys. Rev. D 89, 012004 (2014).

[16] J. P. Lees et al. (BABAR Collaboration), Phys. Rev. D 91, 012003 (2015).

[17] X. L. Wang et al. (Belle Collaboration), Phys. Rev. D 87, 051101(R) (2013).

[18] C. F. Qiao and R. L. Zhu, Phys. Rev. D 89, 074006 (2014).

[19] T. E. Coan et al. (CLEO Collaboration), Phys. Rev. Lett. 96, 162003 (2006). 